\title{
Stereoselective synthesis of alkyl pyranosides on a laboratory scale
}

\author{
Immo Serbian ${ }^{1}$, Erik Prell ${ }^{2}$, Ahmed Al-Harrasi ${ }^{3}$ and René Csuk ${ }^{1, *}$ \\ ${ }^{1}$ Full Address: Martin-Luther-University Halle-Wittenberg, Kurt-Mothes- Str. 2, D-06120 Halle (Saale) \\ ${ }^{2}$ Full Address: University Hospital, Department of Nuclear Medicine, Ernst-Grube-Str. 40, \\ D-06120 Halle (Saale) \\ ${ }^{3}$ Full address: University of Nizwa, Chair of Oman's Medicinal Plants and Marine Natural Products, \\ P.O. Box 33, PC 616, Birkat Al-Mauz, Nizwa, Sultanate of Oman
}

Dedicated to Prof. Amelia P. Rauter on the occasion of her $70^{\text {th }}$ birthday. Ad multos annos!

\begin{abstract}
The one-pot reaction of per-acetylated glycopyranosyl bromides with alcohols in light-protected flask leads to the stereoselective synthesis of deacetylated alkyl pyranosides in good yields.
\end{abstract}

Keywords: alkyl pyranosides; stereoselective synthesis; hexopyranosides.

\section{Introduction}

For a long time, the importance of fatty acids and fatty acid derivatives was limited to their occurrence in mono-, di- and triacylglycerides. The real and greater importance of this class of compounds was finally given by lipidomics. Lipidomics unveils the complexity of the lipidome in metabolic diseases ${ }^{1-5}$. Since then, fatty acid derivatives have increasingly come into the focus of scientific interest, and their importance has increased. For example, glycolipids not only hold many biological functions, including signaling and recognition but also cell adhesion ${ }^{6-8}$.

Recently, it demonstrates that the potential of $\alpha, \beta$-unsaturated fatty acids as inhibitors of the enzymes acetyl- and butyrylcholinesterase ${ }^{9}$, and the antimicrobial and cytotoxic activity of (thio)alkyl hexopyranosides was described ${ }^{10,11}$ These glycolipids are known to destabilize biological membranes resulting most often in antifungal or antibacterial properties ${ }^{12}$. It was shown that especially tetradecyl and hexadecyl $\beta$-D-glucopyranosides hold good antimicrobial activity against some strains of Grampositive bacteria. Their cytotoxicity, however, increases with an increasing chain length of the aglycon ${ }^{10}$. The number of reports dealing with the antimicrobial activity of alkyl glycosides is rare ${ }^{13-16}$.

Today, the selective synthesis of $\alpha$ - or $\beta$-configured glycosides usually does not pose a significant challenge, since a variety of methods exist that allow stereoselective syntheses in good to excellent yields. Unfortunately, these methods have hardly found their

*Corresponding author: René Csuk

Email address: rene.csuk@chemie.uni-halle.de DOI: http://dx.doi.org/10.13171/mjc02003181278rc way into the synthesis of long-chain alkyl glycosides. Within the scope of an extensive study on the cytotoxicity of this class of compounds, we were particularly interested in a rapid stereoselective synthesis of $\beta$-configured glycosides. The method had to be scalable holding reasonable short reaction times, mild conditions and acceptable yields.

\section{Results and discussion}

Our investigations began with the synthesis of methyl glycosides. Fischer glycosidation, the direct reaction of aldoses with boiling methanol in the presence of an acid as a catalyst, usually leads to a mixture of the corresponding $\alpha$ and $\beta$ anomers, and the yields are moderate to low. The reactions proceed fast, but rather time-consuming separations of the anomers cannot be avoided ${ }^{17-20}$. As an alternative chemo-enzymatic glycosidations have been suggested 21-24. These reactions proceed very slowly, and they rarely give acceptable yields ${ }^{25}$. Furthermore, the glycosidases responsible for the creation of the glycosidic bond are not readily commercially available or have to be identified as far as they are yet unknown.

Methanolyses of 2,3,4,6-tetra- $O$-acetyl- $\alpha$-D-glucopyranosyl bromide in the presence of silver salts (e.g. silver(I)triflate) ${ }^{26}$ or mercury salts (Königs-Knorr conditions) ${ }^{27}$ have been widely used ${ }^{28-33}$. However, the use of silver salts for large scale preparations is expensive, and the use of mercury salts raises ecological concern. Additionally, the tin chloride catalysed reaction of 1,2,3,4,6-penta- $O$-acetyl-D-

Received January 23, 2020

Accepted March 3, 2020

Published March 18, 2020 
glucopyranose, however, led to $\beta$-D-glycopyranosides only when $1,2,3,4,6$-penta- $O$-acetyl- $\beta$-Dglucopyranose was used as a starting material ${ }^{34}$. A couple of years ago, the synthesis of methyl $\beta$-D-glucopyranoside from peracetylated $\alpha$-D-glucopyranosyl bromide was re-investigated by H. Weidmann ${ }^{35}$. The scale and the yield were good, but the reported protocol was restricted to the synthesis of methyl $\beta$-D-glucopyranoside. Due to our need in considerable amounts of $\beta$-configurated alkyl glycosides, we became interested in the further development of this procedure.

Our investigations started with the synthesis of methyl $\beta$-D-glucopyranoside (5, Scheme 1) from 2,3,4,6-tetra- $O$-acetyl- $\alpha$-D-glucopyranosyl bromide (4). This compound can be obtained from D-glucose (1) by a two-step synthesis consisting of acetylation of D-glucose (leading to an anomeric mixture of the corresponding per-acetates $\mathbf{2}$ and $\mathbf{3}$ ) followed by their treatment with hydrobromic acid. The reaction of
4 with dry methanol in a light-protected flask at room temperature for four days furnished $85 \%$ of pure 5 being identical with an authentic sample (commercial). Only slight drops of yields were observed upon scaling up of the reaction (up to the amount of ca. $100 \mathrm{~g}$ product prepared in a single flask reaction). The reaction of a mixture of $\mathbf{2}$ and $\mathbf{3}$ with methanol at room temperature in the presence of $\mathrm{BF}_{3} \cdot \mathrm{Et}_{2} \mathrm{O}$ gave a mixture of the anomeric methyl glycosides in $89 \%$ yield.

The reaction of $\mathbf{4}$ with ethanol, propanol, butanol, hexanol, octanol, decanol, dodecanol, tetradecanol and hexadecanol under the same condition (albeit with a prolonged reaction time) gave the corresponding $\beta$-configurated glycosides 6-14. The transformation of $\mathbf{2}$ and $\mathbf{3}$ into $\mathbf{4}$ as well as the conversion of 4 into 5-14 proceeds through an intermediate oxonium ion.
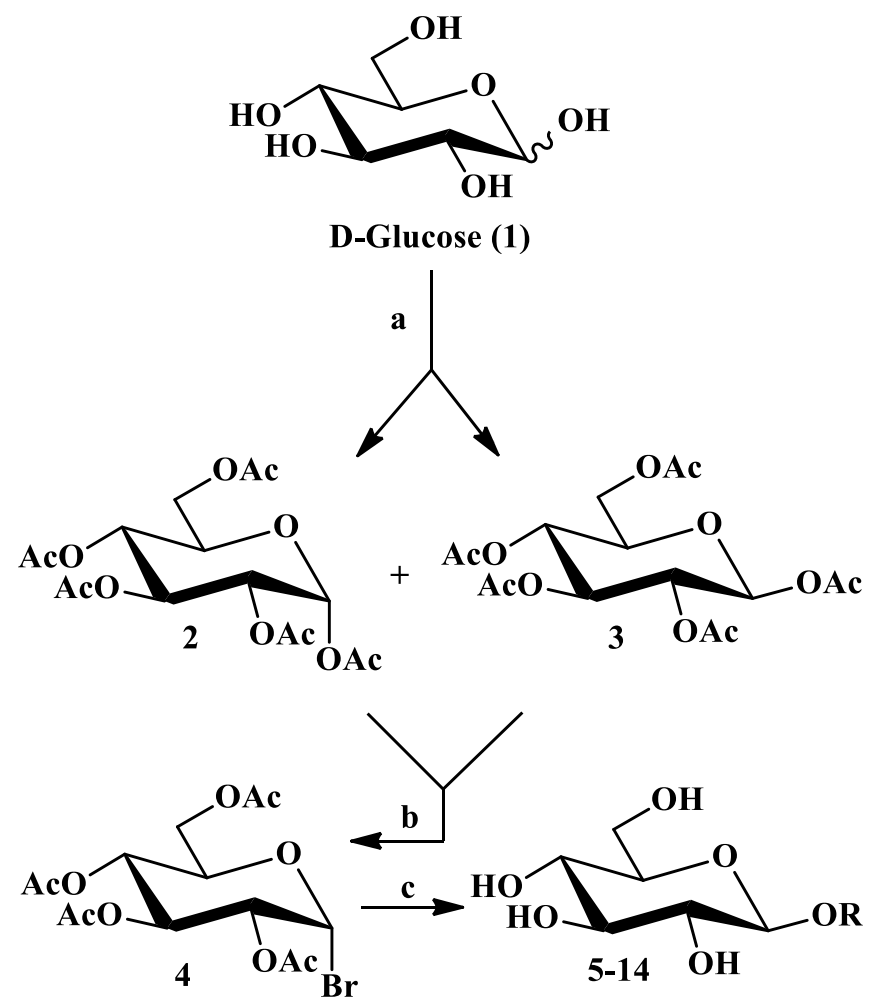

$$
\begin{array}{ll}
5 R=\text { methyl } & 10 R=\text { octyl } \\
6 R=\text { ethyl } & 11 R=\text { decyl } \\
7 R=\text { propyl } & 12 R=\text { dodecyl } \\
8 R=\text { butyl } & 13 R=\text { tetradecyl } \\
9 R=\text { hexyl } & 14 R=\text { hexadecyl }
\end{array}
$$

Scheme 1. Synthesis of $\beta$-configurated glycosides 5-14. Reactions and conditions: a) $\mathrm{Ac}_{2} \mathrm{O}$, pyridine, $3.0 \mathrm{~h}$, $25^{\circ} \mathrm{C}$, quant.; b) $\mathrm{HBr}$ in $\mathrm{AcOH}(33 \%), \mathrm{CHCl}_{3}, 1.0 \mathrm{~h}, 25^{\circ} \mathrm{C}$, quant.; c) $\mathrm{ROH}, 4-7 \mathrm{~d}, 25^{\circ} \mathrm{C}, 56-85 \%$

Similarly, from the reaction of D-mannose (15, Scheme 2) $\beta$-configurated D-mannopyranosyl bromide 18 was obtained in quantitative yield whose reaction with methanol gave a $62 \%$ of methyl $\alpha$-D-mannopyranoside (19). While from D-galactose
(20) finally methyl $\alpha$-D-galactopyranoside (24) was obtained in $56 \%$ yield, and from lactose (15) (via 26 and 27) methyl $\beta$-D-lactoside (28) was accessed in $58 \%$ yield. 
The reactions of the glycosyl bromides with the alcohols have to be performed at room temperature, and bright daylight must be excluded. Heating of the
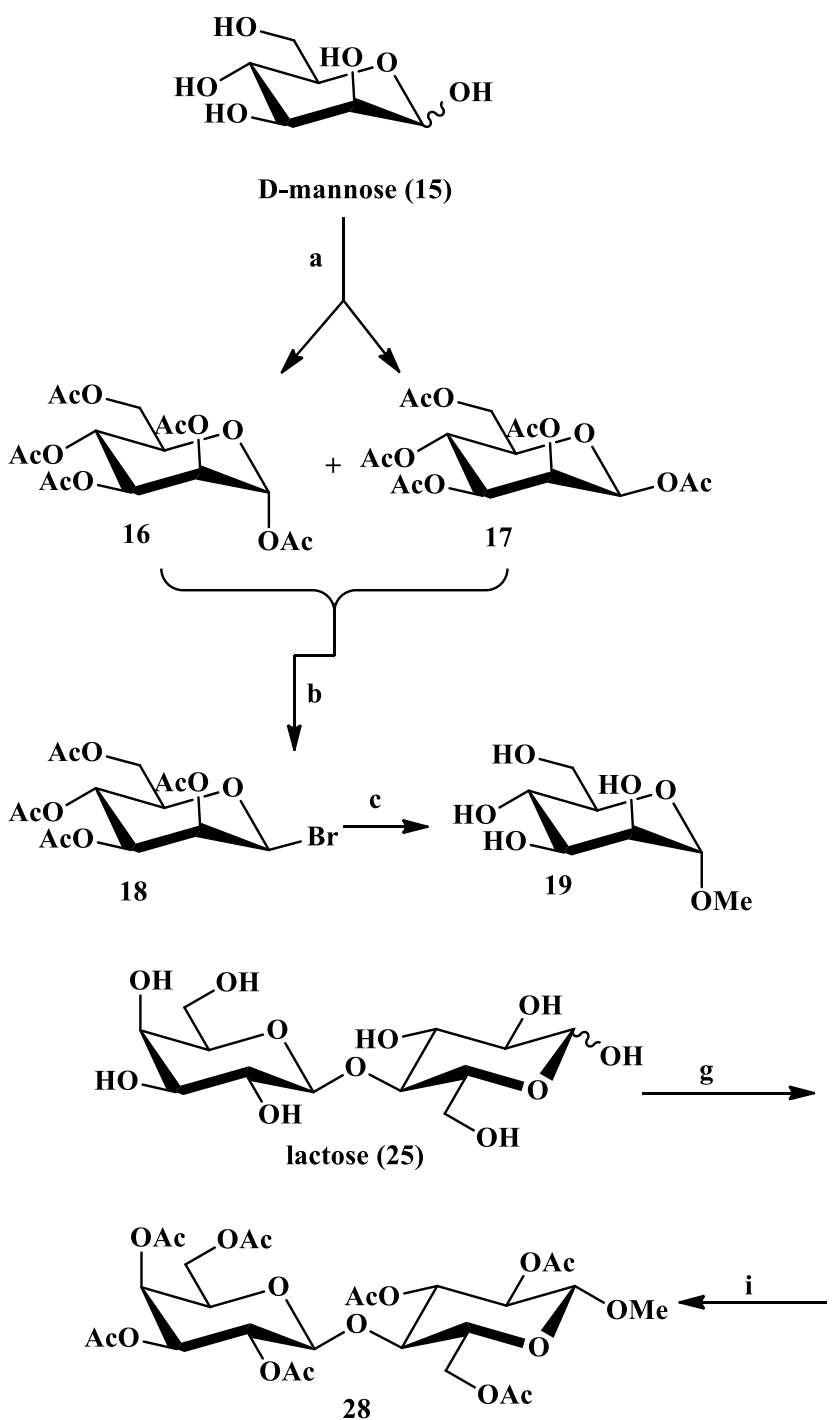

reaction mixtures resulted in the formation of mixtures of anomers while standing in bright daylight led to the decomposition of the glycosyl bromides.
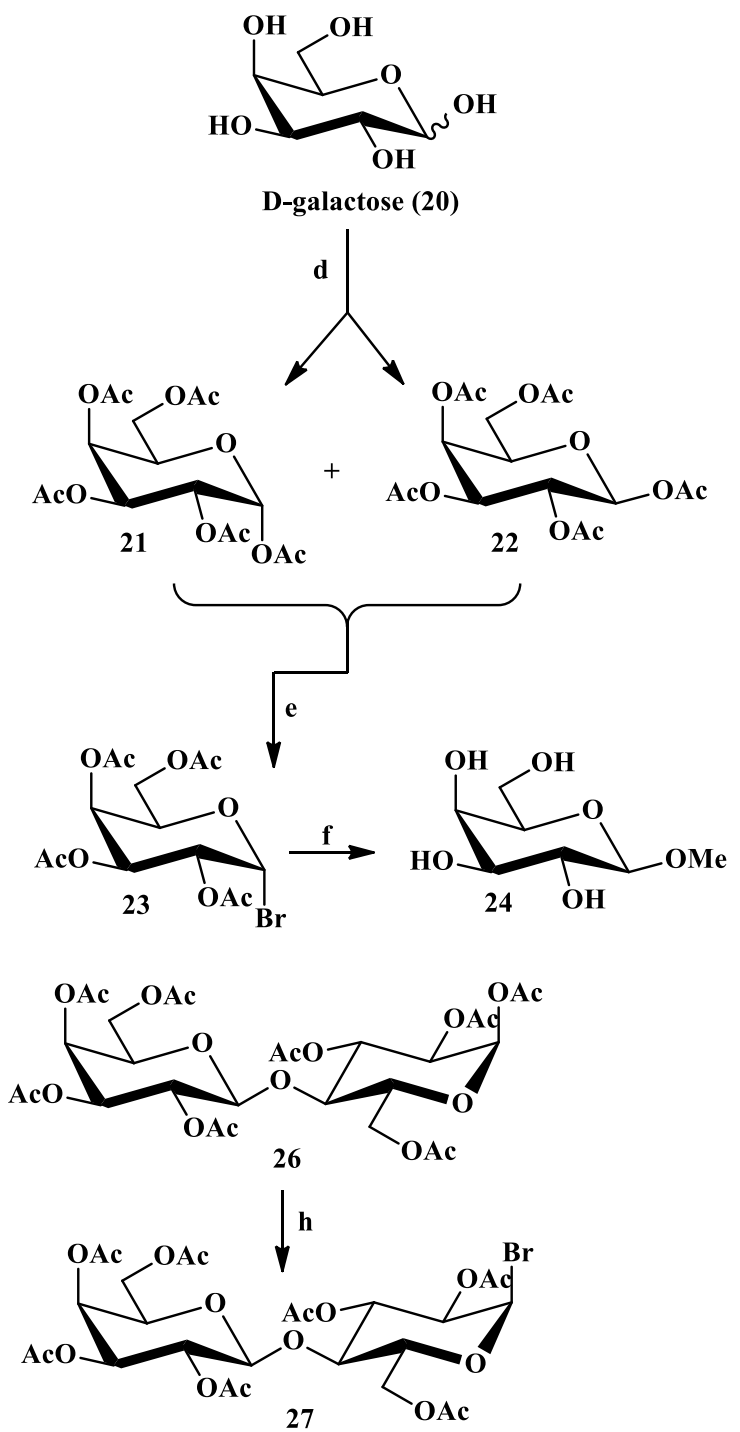

Scheme 2. Synthesis of methyl glycosides 19, 24 and $\mathbf{2 8}$ derived from D-mannose (15), D-galactose (20) and lactose (25), respectively: reactions and conditions: a) d) g) $\mathrm{Ac}_{2} \mathrm{O}$, pyridine, $3 \mathrm{~h}, 25^{\circ} \mathrm{C}$, quant.; b) e) h) $\mathrm{HBr}$ in $\mathrm{AcOH}\left(33 \%\right.$ ), $\mathrm{CHCl}_{3}, 1 \mathrm{~h}, 25^{\circ} \mathrm{C}$, quant.; c) f) i) $\mathrm{MeOH}, 4 \mathrm{~d}, 25^{\circ} \mathrm{C}, 62 \%$ (of 19), $56 \%$ (of 24), 58\% (of 28)

\section{Conclusion}

The reaction of glycosyl bromides in the presence of the corresponding alcohols leads to the respective glycosides in good yields. Starting from D-glucose, D-galactose and lactose, the $\beta$-configured glycosides are stereoselectively obtained. While the corresponding $\alpha$-configured product is obtained from D-mannose. The method described here is simple, can be easily scaled up for more considerable preparations and offers the advantage of not requiring additional metal salt or base catalysis.

\section{Experimental}

Instrumentation as previously ${ }^{9}$ reported. The purity of the compounds was determined by HPLC (Merck-Hitachi LaChrom D-7000 HPLC-DAD/RI system, column, Aminex HPX-87P; 300x7.8 mm; $60{ }^{\circ} \mathrm{C} ; \mathrm{H}_{2} \mathrm{O}$; flow $\left.0.5 \mathrm{~mL} / \mathrm{min}\right)>98 \%$. ${ }^{1} \mathrm{H}$ and ${ }^{13} \mathrm{C}$ NMR data for known compounds were as reported.

\section{1,2,3,4,6-Penta- $O$-acetyl- $\alpha$-D-glucopyranose} and $1,2,3,4,6$-penta- $O$-acetyl- $\beta$-D-glucopyranose (3)

Acetylation of anhydrous D-glucose $(\mathbf{1}, 30.0 \mathrm{~g}, 166.5 \mathrm{mmol})$ in dry pyridine $(110 \mathrm{~mL})$ with $\mathrm{Ac}_{2} \mathrm{O}(109 \mathrm{~mL}, 1.17 \mathrm{~mol})$ for 5 hours at $25^{\circ} \mathrm{C}$ followed by usual aqueous workup, furnished a mixture of 2 and $3(65.0 \mathrm{~g}, 100 \%)$ being pure enough for the next step. One gram of this mixture was subjected to chromatography (silica gel, hexane/ethyl acetate, 5:3) to afford pure samples. 
Data for 2: m.p. $109-111^{\circ} \mathrm{C}$ (lit.: ${ }^{36} 111-112^{\circ} \mathrm{C}$ ); $[\alpha]_{\mathrm{D}}$ $=+76.3^{\circ}\left(c=0.6, \mathrm{CHCl}_{3}\right)$ (lit.: ${ }^{37}[\alpha]_{\mathrm{D}}=+104^{\circ}$ $\left.\left(c=0.5, \mathrm{CHCl}_{3}\right)\right)$.

Data for 3: m.p. $128-130^{\circ} \mathrm{C}$ (lit.: $\left.{ }^{38} 131-132^{\circ} \mathrm{C}\right)$; $[\alpha]_{\mathrm{D}}$ $=+4.6^{\circ}\left(c=0.5, \mathrm{CHCl}_{3}\right)\left(\right.$ lit.: ${ }^{39}[\alpha]_{\mathrm{D}}=+3.8^{\circ}(c=1.0$, $\left.\mathrm{CHCl}_{3}\right)$ ).

\section{2,3,4,6-Tetra- $O$-acetyl- $\alpha$-D-glucopyranosyl bromide (4)}

To an ice-cold solution of 2/3 (65.0 g, $166.5 \mathrm{mmol})$ in dry $\mathrm{CHCl}_{3}(200 \mathrm{~mL})$, hydrobromic acid $(33 \%$ in $\mathrm{AcOH}$ ) was slowly added. After stirring for $20 \mathrm{~min}$, the mixture was stirred for another hour at $25^{\circ} \mathrm{C}$, cooled to $5^{\circ} \mathrm{C}$, and water $(200 \mathrm{~mL})$ was added. The organic layer was washed [water $(3 \times 100 \mathrm{~mL})$, $\mathrm{NaHCO}_{3}$ (satd., $2 \times 100 \mathrm{~mL}$ )], dried $\left(\mathrm{Na}_{2} \mathrm{SO}_{4}\right)$, and the volatiles were removed under diminished pressure. Column chromatography (silica gel, hexane/ethyl acetate, 5:3) afforded $4(68.47 \mathrm{~g}, 100 \%)$. The material was directly used for the next reactions. Re-crystallization from diisopropyl ether gave an analytical sample. Re-crystallized material can be stored in the dark over $\mathrm{KOH}$ for several months while impure material readily becomes brown to black under air and light; m.p. $87-89^{\circ} \mathrm{C}$ (lit.: ${ }^{40} 88-89^{\circ} \mathrm{C}$ ); $[\alpha]_{\mathrm{D}}+197.3^{\circ}\left(c=1.8, \mathrm{CHCl}_{3}\right)$ (lit.: ${ }^{40}+197^{\circ}$ $\left.\left(c=2, \mathrm{CHCl}_{3}\right)\right)$.

\section{Methyl $\boldsymbol{\beta}$-D-glucopyranoside (5)}

Stirring of a solution of $4(68.5 \mathrm{~g}, 166.5 \mathrm{mmol})$ in dry methanol $(400 \mathrm{~mL})$ in a light-protected flask (black foil) at $25^{\circ} \mathrm{C}$ for 4 days (for the reaction utilizing long-chain alkanols reaction times of up to 7 days were used for completion of the reaction; TLC checked the progress) followed by removal of the volatiles under reduced pressure and crystallization from methanol/ethanol (1:3) afforded 5 (27.2 g, 85\%); m.p. $\quad 110-111^{\circ} \mathrm{C}$ (lit.: ${ }^{41} 112-113^{\circ} \mathrm{C}$ ); m.m.p. $110-111^{\circ} \mathrm{C} ; \quad[\alpha]_{\mathrm{D}}=-32.7^{\circ}(c=0.4, \mathrm{MeOH})$ (lit.: ${ }^{42}[\alpha]_{\mathrm{D}}=-30.3^{\circ}\left(c=2, \mathrm{H}_{2} \mathrm{O}\right)$. Scaling up of the reaction ( $274 \mathrm{~g}$ starting material) gave a slight drop of yield $(76 \%$ after work-up by chromatography (silica gel, methanol/ethyl acetate 10:90 $\rightarrow$ methanol/ethyl acetate 20:80) instead of crystallization.

\section{Ethyl $\boldsymbol{\beta}$-D-glucopyranoside (6)}

As described above, 4 (6.85 g, $16.6 \mathrm{mmol})$ gave $6(2.86 \mathrm{~g}, 83 \%)$; m.p. $81-83^{\circ} \mathrm{C}$ (lit.: ${ }^{43} 82-84^{\circ} \mathrm{C}$ ); $[\alpha]_{\mathrm{D}}=-33.0^{\circ}(c=0.8, \mathrm{MeOH})\left(\right.$ lit.: ${ }^{43}[\alpha]_{\mathrm{D}}=-28.5^{\circ}$ $(c=1.0, \mathrm{MeOH}))$.

\section{Propyl $\boldsymbol{\beta}$-D-glucopyranoside (7)}

As described above, 4 (6.85 g, $16.6 \mathrm{mmol})$ gave 7 (2.95 g, 80\%); m.p. $97-100^{\circ} \mathrm{C}$ (lit.: ${ }^{44} 101-102^{\circ} \mathrm{C}$ ); $[\alpha]_{\mathrm{D}}=-37.4^{\circ}(c=0.9, \mathrm{MeOH}) \quad\left(\right.$ lit.: ${ }^{45}[\alpha]_{\mathrm{D}}=-44.5^{\circ}$ $(c=1.16, \mathrm{MeOH}))$.

\section{Butyl $\boldsymbol{\beta}$-D-glucopyranoside (8)}

As described above, 4 (6.85 g, $16.6 \mathrm{mmol})$ gave 8
(2.98 g, 76\%); m.p. $83-85^{\circ} \mathrm{C}$ (lit.: ${ }^{46} 81-82^{\circ} \mathrm{C}$ ); $[\alpha]_{\mathrm{D}}=-35.9^{\circ}(c=0.4, \mathrm{MeOH})\left(\right.$ lit.: ${ }^{46}[\alpha]_{\mathrm{D}}=-35.2^{\circ}$ $(c=1.4, \mathrm{MeOH}))$.

\section{Hexyl $\beta$-D-glucopyranoside (9)}

As described above, 4 (6.85 g, $16.6 \mathrm{mmol})$ gave 8 (3.11 g, 71\%); m.p. $88-90^{\circ} \mathrm{C}$ (lit.: ${ }^{47} 88-89^{\circ} \mathrm{C}$ ); $[\alpha]_{\mathrm{D}}=-33.4^{\circ}(c=0.7, \mathrm{MeOH})\left(\right.$ lit.: ${ }^{45}[\alpha]_{\mathrm{D}}=-33.9^{\circ}$ $(c=0.9, \mathrm{MeOH}))$.

\section{Octyl $\boldsymbol{\beta}$-D-glucopyranoside (10)}

As described above, $4(6.85 \mathrm{~g}, 16.6 \mathrm{mmol})$ gave $\mathbf{1 0}$ (3.25 g, 67\%); m.p. $73-77^{\circ} \mathrm{C}$ (lit.: ${ }^{48} 61.5-62.5{ }^{\circ} \mathrm{C}$ ); $[\alpha]_{\mathrm{D}}=-39.8^{\circ}\left(c=0.6, \mathrm{H}_{2} \mathrm{O}\right)\left(\right.$ lit.: ${ }^{49}[\alpha]_{\mathrm{D}}=-39.8^{\circ}$ $\left.\left(c=0.6, \mathrm{H}_{2} \mathrm{O}\right)\right)$.

\section{Decyl $\beta$-D-glucopyranoside (11)}

As described above (in the presence of $100 \mathrm{~mL}$ dichloromethane), 4 (6.85 g, $16.6 \mathrm{mmol})$ gave 11 (3.67 g, 69\%); m.p. $130-135^{\circ} \mathrm{C}$ (lit.: ${ }^{48} 135.6^{\circ} \mathrm{C}$ ); $[\alpha]_{\mathrm{D}}=-27.8^{\circ}(c=0.6, \mathrm{MeOH})\left(\right.$ lit.: ${ }^{50}[\alpha]_{\mathrm{D}}=-27.8^{\circ}$ $(c=0.7, \mathrm{MeOH}))$.

\section{Dodecyl $\beta$-D-glucopyranoside (12)}

As described above (in the presence of $100 \mathrm{~mL}$ dichloromethane), $4(6.85 \mathrm{~g}, 16.6 \mathrm{mmol})$ gave 12 (3.70 g, 64\%); m.p. $138-142^{\circ} \mathrm{C}$ (lit.: $\left.{ }^{48} 144-145^{\circ} \mathrm{C}\right)$; $[\alpha]_{\mathrm{D}}=-23.1^{\circ}(c=0.4, \mathrm{MeOH})\left(\right.$ lit.: ${ }^{50}[\alpha]_{\mathrm{D}}=-24.7^{\circ}$ $(c=0.5, \mathrm{MeOH}))$.

\section{Tetradecyl $\beta$-D-glucopyranoside (13)}

As described above (in the presence of $100 \mathrm{~mL}$ dichloromethane), 4 (6.85 g, $16.6 \mathrm{mmol})$ gave 13 (3.56 g, 57\%); m.p. $147-151^{\circ} \mathrm{C}$ (lit.: ${ }^{51} 151.5^{\circ} \mathrm{C}$ ); $[\alpha]_{\mathrm{D}}=-30.4^{\circ}(c=0.3, \mathrm{MeOH})\left(\right.$ lit.: ${ }^{52}[\alpha]_{\mathrm{D}}=-32.4^{\circ}$ $(c=0.6, \mathrm{MeOH}))$.

\section{Hexadecyl $\boldsymbol{\beta}$-D-glucopyranoside (14)}

As described above (in the presence of $100 \mathrm{~mL}$ dichloromethane), 4 (6.85 g, $16.6 \mathrm{mmol})$ gave 13 (3.90 g, 58\%); m.p. = $138-141^{\circ} \mathrm{C}$ (lit.: ${ }^{52} 145^{\circ} \mathrm{C}$ ); $[\alpha]_{\mathrm{D}}=-19.9^{\circ}\left(c=0.2, \mathrm{CHCl}_{3}\right)\left(\right.$ lit.: ${ }^{53}[\alpha]_{\mathrm{D}}=-16.2^{\circ}$ $\left.\left(c=0.5, \mathrm{CHCl}_{3}\right)\right)$.

\section{1,2,3,4,6-Penta- $O$-acetyl- $\alpha$-D-mannopyranose (16) and $1,2,3,4,6$-penta- $O$-acetyl- $\beta$-D-mannopyranose} (17)

As described above, from D-mannose $(\mathbf{1 5}, 30.0 \mathrm{~g}, 166.5 \mathrm{mmol})$ a mixture of syrupy $\mathbf{1 6} / \mathbf{1 7}$ (68.5 g, 100\%). Chromatography gave analytically pure compounds:

Data for 16: m.p. $74-76^{\circ} \mathrm{C}$ (lit.: ${ }^{54} 75-76^{\circ} \mathrm{C}$ ); $[\alpha]_{\mathrm{D}}=+58.0^{\circ}\left(c=0.75, \mathrm{CHCl}_{3}\right)\left(\right.$ lit.: ${ }^{54}[\alpha]_{\mathrm{D}}+56.8^{\circ}$ $\left.\left(c=2.0, \mathrm{CHCl}_{3}\right)\right)$.

Data for 17: m.p. $115-118^{\circ} \mathrm{C}$ (lit.: $\left.{ }^{55} 117-118^{\circ} \mathrm{C}\right) ;[\alpha]_{\mathrm{D}}$ $-83.4^{\circ}\left(c=0.32, \mathrm{CHCl}_{3}\right)$; lit.: ${ }^{56}[\alpha]_{\mathrm{D}}-85.5^{\circ}$ $\left.\left(c=1.5, \mathrm{CHCl}_{3}\right)\right)$. 


\section{$2,3,4,6$-Tetra- $O$-acetyl- $\beta$-D-mannopyranosyl bromide (18)}

As described above, 16/17 (65.0 g, $189.9 \mathrm{mmol})$ gave syrupy 18 (68.5 g, 100\%). Chromatography yielded an analytically pure compound:

m.p. $69-72^{\circ} \mathrm{C} ;[\alpha]_{\mathrm{D}}+52.8^{\circ}\left(c=0.59, \mathrm{CHCl}_{3}\right)$;

${ }^{1} \mathrm{H}$ NMR $\left(500 \mathrm{MHz}, \mathrm{CDCl}_{3}\right): \delta=6.29(\mathrm{~d}, 1 \mathrm{H}, \mathrm{J}=1.6$ $\mathrm{Hz}, 1-\mathrm{H}), 5.71(\mathrm{dd}, 1 \mathrm{H}, \mathrm{J}=3.4,10.2 \mathrm{~Hz}, 3-\mathrm{H}), 5.44$ (dd, $1 \mathrm{H}, \mathrm{J}=1.6,3.4 \mathrm{~Hz}, 2-\mathrm{H}), 5.37$ (dd, $1 \mathrm{H}, \mathrm{J}=10.1$, $10.2 \mathrm{~Hz}, 4-\mathrm{H}), 4.32$ (dd, 1H, J= 4.9, $12.5 \mathrm{~Hz}, 6-\mathrm{H}_{\mathrm{A}}$ ), $4.22(\mathrm{ddd}, 1 \mathrm{H}, \mathrm{J}=2.2,4.9,10.1 \mathrm{~Hz}, 5-\mathrm{H}), 4.14(\mathrm{dd}$, $\left.1 \mathrm{H}, \mathrm{J}=2.2,12.5 \mathrm{~Hz}, 6-\mathrm{H}_{\mathrm{B}}\right), 2.17,2.10,2.07,2.00$ (each s, 3H, Me) ppm;

${ }^{13} \mathrm{C} \mathrm{NMR}\left(125 \mathrm{MHz}, \mathrm{CDCl}_{3}\right): \delta=170.5,169,6,169.5$, 169.4 (each CO), 83.0 (C-1), 72.8 (C-5), 72.1 (C-4), 67.9 (C-3), 65.3 (C-2), 61.4 (C-6), 20.7, 20.6, 20.5, 20.4 (each Me) ppm;

MS (ESI, MeOH): m/z $(\%)=331.1\left([\mathrm{M}-\mathrm{Br}]^{+}, 16\right)$, $427.9\left(\left[\mathrm{M}\left({ }^{79} \mathrm{Br}\right)+\mathrm{NH}_{4}\right]^{+}, 18\right), 429.9\left(\left[\mathrm{M}\left({ }^{81} \mathrm{Br}\right)+\right.\right.$ $\left.\mathrm{NH}_{4}\right]^{+} 7,432.9\left(\left[\mathrm{M}\left({ }^{79} \mathrm{Br}\right)+\mathrm{Na}\right]^{+}, 99\right), 434.8\left(\left[\mathrm{M}\left({ }^{81} \mathrm{Br}\right)\right.\right.$ $\left.+\mathrm{Na}]^{+}, 100\right)$;

analysis calcd for $\mathrm{C}_{14} \mathrm{H}_{19} \mathrm{BrO}_{9}$ (411.20): C 40.89, H 4.66; found: C 40.63, H 4.85.

\section{Methyl $\alpha$-D-mannopyranoside (19)}

As described above, 18 (68.5 g, $166.5 \mathrm{mmol})$ gave $\mathbf{1 9}$ (20.1 g, 62\%); m.p. $189-191^{\circ} \mathrm{C}$ (lit.: ${ }^{57} 194^{\circ} \mathrm{C}$ ); $[\alpha]_{\mathrm{D}}=+81.0^{\circ}\left(c=0.38, \mathrm{H}_{2} \mathrm{O}\right)\left(\right.$ lit.: ${ }^{57}[\alpha]_{\mathrm{D}}=+82.5^{\circ}$ $\left.\left(c=1.3, \mathrm{H}_{2} \mathrm{O}\right)\right)$.

\section{1,2,3,4,6-Penta- $O$-acetyl- $\alpha$-D-galactopyranose (21) and $1,2,3,4,6$-penta- $O$-acetyl- $\beta$-D-galactopyranose} (22)

As described above, D-galactose $(\mathbf{2 0}, 30.0 \mathrm{~g}, 166.6$ mmol) gave a syrupy mixture of $\mathbf{2 1 / 2 2}(68.5 \mathrm{~g}, 100 \%)$. Chromatography yielded analytically pure compounds:

Data for 21: m.p. $92-95^{\circ} \mathrm{C}$ (lit.: $58 \quad 95-96^{\circ} \mathrm{C}$ ); $[\alpha]_{\mathrm{D}}+103.7^{\circ}\left(c=0.55, \mathrm{CHCl}_{3}\right)\left(\right.$ lit.: ${ }^{58}[\alpha]_{\mathrm{D}}+106.8^{\circ}$ $\left.\left(c=3.01, \mathrm{CHCl}_{3}\right)\right)$.

Data for 22: m.p. $140-143^{\circ} \mathrm{C}$ (lit: ${ }^{59} 143-144^{\circ} \mathrm{C}$ ); $[\alpha]_{\mathrm{D}}+26.0^{\circ}\left(c=0.4, \mathrm{CHCl}_{3}\right)\left(\right.$ lit.: ${ }^{60}[\alpha]_{\mathrm{D}}+27.1^{\circ}(c=$ $\left.1.03, \mathrm{CHCl}_{3}\right)$ ).

\section{2,3,4,6-Tetra- $O$-acetyl- $\alpha$-D-galactoyranosyl bromide (23)}

As described above, 21/22 (65.0 g, $189.9 \mathrm{mmol})$ gave syrupy 23 (68.5 g, 100\%). Chromatography yielded an analytically pure compound: m.p. $84-86^{\circ} \mathrm{C}$ (lit.: $\left.{ }^{61} 84-85^{\circ} \mathrm{C}\right) ;[\alpha]_{\mathrm{D}}+209.8^{\circ}\left(c=0.54, \mathrm{CHCl}_{3}\right)$ (lit.: ${ }^{62}[\alpha]_{\mathrm{D}}=+212^{\circ}\left(c=1, \mathrm{CHCl}_{3}\right)$ ).

\section{Methyl $\boldsymbol{\beta}$-D-galactopyranoside (24)}

As described above, 23 (68.5 g, $166.5 \mathrm{mmol})$ gave $\mathbf{2 4}$ (18.1 g, 56\%); m.p. $171-173^{\circ} \mathrm{C}$ (lit.: ${ }^{63} 175-178^{\circ} \mathrm{C}$ ); $[\alpha]_{\mathrm{D}}=-13.5^{\circ}(c=0.65, \mathrm{MeOH})\left(\right.$ lit.: ${ }^{63}[\alpha]_{\mathrm{D}}=-15.5^{\circ}$ $(c=1.0 \mathrm{MeOH}))$.

\section{$1,2,3,6,2^{\prime}, 3^{\prime}, 4^{\prime}, 6^{\prime}$-Octa-O-acetyl- $\alpha$-lactose (26)}

As described above, lactose $(\mathbf{2 5}, 30.0 \mathrm{~g}, 87.7 \mathrm{mmol})$ gave syrupy 26 (59.5 g, 100\%). An analytical sample showed: m.p. $135-138^{\circ} \mathrm{C}$ (lit.: ${ }^{64} 140-143^{\circ} \mathrm{C}$ ); $[\alpha]_{\mathrm{D}}=+94.1^{\circ}\left(c=0.73, \mathrm{CHCl}_{3}\right)\left(\right.$ lit.: ${ }^{65}[\alpha]_{\mathrm{D}}=+99^{\circ}$ $\left(c=2, \mathrm{CH}_{2} \mathrm{Cl}_{2}\right)$;

${ }^{1} \mathrm{H}$ NMR $\left(500 \mathrm{MHz}, \mathrm{CDCl}_{3}\right): \delta=6.25(\mathrm{~d}, 1 \mathrm{H}, \mathrm{J}=3.7$ $\mathrm{Hz}, 1-\mathrm{H}), 5.45(\mathrm{dd}, 1 \mathrm{H}, \mathrm{J}=9.4,10.2 \mathrm{~Hz}, 3-\mathrm{H}), 5.35$ (dd, $\left.1 \mathrm{H}, \mathrm{J}=1.0,3.4 \mathrm{~Hz}, 4^{\prime}-\mathrm{H}\right), 5.12(\mathrm{dd}, 1 \mathrm{H}, \mathrm{J}=7.9$, $\left.10.4 \mathrm{~Hz}, 2^{\prime}-\mathrm{H}\right), 5.00$ (dd, $\left.1 \mathrm{H}, \mathrm{J}=3.7,10.2 \mathrm{~Hz}, 2-\mathrm{H}\right)$, $4.96\left(\mathrm{dd}, 1 \mathrm{H}, \mathrm{J}=3.4,10.4 \mathrm{~Hz}, 3^{\prime}-\mathrm{H}\right), 4.48$ (d, 1H, J = $\left.7.9 \mathrm{~Hz}, 1^{\prime}-\mathrm{H}\right), 4.44$ (dd, $\left.1 \mathrm{H}, \mathrm{J}=2.0,12.2 \mathrm{~Hz}, 6^{\prime}-\mathrm{H}_{\mathrm{A}}\right)$, 4.16-4.06 (m, 3H, 6- $\left.\mathrm{H}_{\mathrm{A}}, 6-\mathrm{H}_{\mathrm{B}}, 6^{\prime}-\mathrm{H}_{\mathrm{B}}\right), 4.00$ (ddd, $1 \mathrm{H}$, $\mathrm{J}=1.9,4.1,10.0 \mathrm{~Hz}, 5-\mathrm{H}), 3.87$ (ddd, $1 \mathrm{H}, \mathrm{J}=2.0,6.0$, $7.5 \mathrm{~Hz}, 5$ '-H), 3.81 (dd, $1 \mathrm{H}, \mathrm{J}=9.4,10.0 \mathrm{~Hz}, 4-\mathrm{H})$, $2.17,2.15,2.12,2.06,2.05,2.03,2.00,1.96$ (each s, 3 $\mathrm{H}, \mathrm{OAc}) \mathrm{ppm}$;

${ }^{13} \mathrm{C} \mathrm{NMR}\left(125 \mathrm{MHz}, \mathrm{CDCl}_{3}\right): \delta=170.3,170.2,170.1$, 170.0, 169.9, 169.5, 169.1, 168.9 (each CO), 101.2 (C-1'), 89.0 (C-1), 75.8 (C-4), 71.0 (C-3'), 70.7 (C3), 69.6 (C-2), 6.4 (C-2'), 66.6 (C-4'), 61.4 (C-6'), 60.8 (C-6), 20.9, 20.8, 20.7, 20.6, 20.5 20.4, 20.3, 20.3 (each $\mathrm{CH}_{3}$ );

MS (ESI, MeOH): m/z $(\%)=691.1\left(\left[\mathrm{M}+\mathrm{NH}_{4}\right]^{+}, 44\right)$, $701.1\left([\mathrm{M}+\mathrm{Na}]^{+}, 100\right), 1378.3\left(\left[\mathrm{M}_{2}+\mathrm{Na}\right]^{+}, 26 \%\right)$; analysis calcd for $\mathrm{C}_{28} \mathrm{H}_{38} \mathrm{O}_{19}$ (678.59): $\mathrm{C} 49.56, \mathrm{H}$ 5.64; found: C 49.37, H 5.81.

\section{2,3,6,2',3',4'6'-Hepta- $O$-acetyl- $\alpha$-lactopyranosyl} bromide (27)

As described above, 26 (59.5 g, $87.6 \mathrm{mmol})$ gave 27 (61.3 g, 100\%). Chromatography yielded an analytically pure compound: m.p. ${ }^{65} 138-141^{\circ} \mathrm{C}$ (lit.: $\left.143-144^{\circ} \mathrm{C}\right) ;[\alpha]_{\mathrm{D}}=+103.6^{\circ}\left(c=0.53, \mathrm{CHCl}_{3}\right)$ (lit.: ${ }^{66} 110.6^{\circ}\left(c=0.4, \mathrm{CHCl}_{3}\right)$ ).

\section{Methyl $\boldsymbol{\beta}$-lactoside (28)}

As described above, 27 (61.3 g, $87.6 \mathrm{mmol})$ gave $28(18.1 \mathrm{~g}, 58 \%)$;

m.p. $203-205^{\circ} \mathrm{C}$ (lit.: ${ }^{67} 205^{\circ} \mathrm{C}$ ); $[\alpha]_{\mathrm{D}}=-13.0^{\circ}$ $(c=0.5, \mathrm{MeOH})\left(\right.$ lit.: $\left.{ }^{68}[\alpha]_{\mathrm{D}}=+3.6^{\circ}\left(c=1.4, \mathrm{H}_{2} \mathrm{O}\right)\right)$; ${ }^{1} \mathrm{H}$ NMR $\left(500 \mathrm{MHz}, \mathrm{CD}_{3} \mathrm{OD}\right): \delta=4.35(\mathrm{~d}, 1 \mathrm{H}, \mathrm{J}=7.6$ $\mathrm{Hz}, 1-\mathrm{H}), 4.19$ (d, $1 \mathrm{H}, \mathrm{J}=7.8 \mathrm{~Hz}, 1$ '-C), 3.90 (dd, $1 \mathrm{H}$, $\left.\mathrm{J}=2.5,12.1 \mathrm{~Hz}, 6^{\prime}-\mathrm{H}_{\mathrm{A}}\right), 3.84(\mathrm{ddm} 1 \mathrm{H}, \mathrm{J}=4.2,12.1$ $\left.\mathrm{Hz}, 6^{\prime}-\mathrm{H}_{\mathrm{B}}\right), 3.80$ (dd, J = 0.9, 3.2 Hz, 4'-H), 3.77 (dd, $\left.1 \mathrm{H}, \mathrm{J}=7.5,11.4 \mathrm{~Hz}, 6-\mathrm{H}_{\mathrm{A}}\right), 3.69(\mathrm{dd}, 1 \mathrm{H}, \mathrm{J}=4.6,11.4$ $\mathrm{Hz}, 6-\mathrm{H}_{\mathrm{B}}$ ), 3.59-3.53 (m, 4H, 2-H, 3H, 4-H, 5-H), 3.52 (s, 3H, OMe), 3.47 (dd, 1J, J = 3.2, $9.5 \mathrm{~Hz}, 3$ '-H), 3.39 (ddd, $1 \mathrm{H}, \mathrm{J}=0.9,2.5,4.2 \mathrm{~Hz}, 5$ ' $-\mathrm{C}), 3.22$ (dd, $1 \mathrm{H}, \mathrm{J}=$ 7.8, $9.5 \mathrm{~Hz}, 2$ '-C) ppm;

${ }^{13} \mathrm{C}$ NMR (125 MHz, $\left.\mathrm{CD}_{3} \mathrm{OD}\right): \delta=103.8\left(\mathrm{C}-1^{\prime}\right)$, 103.7 (C-1), 79.2 (C-5), 75.7 (C-2'), 75.0 (C-2), 74.9 (C-4), 73.4 (C-3'), 73.3 (C-3), 71.1 (C-5'), 68.9 (C-4'), 61.0 (C-6'), 60.5 (C-6), 55.9 (OMe) ppm; MS (ESI, $\mathrm{MeOH}): \mathrm{m} / \mathrm{z}(\%)=357.1\left([\mathrm{M}+\mathrm{H}]^{+}, 2\right)$, $379.3\left([\mathrm{M}+\mathrm{Na}]^{+}, 14\right), 734.9\left([2 \mathrm{M}+\mathrm{Na}]^{+}, 100\right)$; analysis calcd for $\mathrm{C}_{1} 3 \mathrm{H}_{24} \mathrm{O}_{11}$ (356.32): $\mathrm{C} 43.82, \mathrm{H}$ 6.79; found: C 43.61, H 6.94.

\section{Acknowledgments}

We like to thank Dr. D. Ströhl and his team for the NMR spectra and Dr. R. Kluge for measuring the MS spectra, and to V. Simon for taking the IR spectra as 
well as for the determination of the optical rotations. $\mathrm{S}$. Ludwig measured the micro-analyses.

\section{References}

1- J. Bestard-Escalas, A. Maimó-Barceló, K. PérezRomero, D. H. Lopez, G. Barceló-Coblijn, Ins and Outs of Interpreting Lipidomic Results, J. Mol. Biol., 2019, 43, 5039-5062.

2- M. Cuperlovic-Culf, A. Badhwar, Recent advances from metabolomics and lipidomics application in alzheimer's disease inspiring drug discovery, Expert Opin. Drug Discovery, 2020, 15, 319-331.

3- M. M. Khan, O. Ernst, N. P. Manes, B. L. Oyler, I. D. C. Fraser, D. R. Goodlett, A. Nita-Lazar, Multi-Omics Strategies Uncover Host-Pathogen Interactions, ACS Infect. Dis., 2019, 5, 493-505.

4- T. Porta Siegel, K. Ekroos, S. R. Ellis, Reshaping Lipid Biochemistry by Pushing Barriers in Structural Lipidomics, Angew. Chem., Int. Ed., 2019, 58, 6492-6501.

5- V. V. Smirnov, E. A. Egorenkov, T. N. Myasnikova, A. E. Petukhov, V. I. Gegechkori, A. M. Sukhanova, G. V. Ramenskaya, Lipidomic analysis as a tool for identifying susceptibility to various skin diseases, MedChemComm, 2019, 10, 1871-1874.

6- A. d. J. Cortés-Sanchez, H. Hernández-Sánchez, M. E. Jaramillo-Flores, Biological activity of glycolipids produced by microorganisms: New trends and possible therapeutic alternatives, Microbiol. Res., 2013, 168, 22-32.

7- Y. Ishibashi, A. Kohyama-Koganeya, Y. Hirabayashi, New insights on glucosylated lipids: Metabolism and functions, Biochim. Biophys. Acta, Mol. Cell Biol. Lipids, 2013,1831, 1475-1485.

8- M. Schombs, J. Gervay-Hague, Glycochemistry: overview and progress, Glycochemical Synthesis: Strategies and Applications, 2016, 1-34.

9- A. Loesche, J. Wiemann, Z. Al Halabi, J. Karasch, W. Sippl, R. Csuk, Unexpected AChE inhibitory activity of $(2 E) \alpha, \beta$-unsaturated fatty acids, Bioorg. Med. Chem. Lett., 2018, 28, 3315-3319.

10-P. Dzubak, S. Gurska, B. K., D. Uhrikova, N. Kanjakova, S. Combet, T. Klunda, M. Kolar, M. Hajduch, M. Polakova, Antimicrobial and cytotoxic activity of (thio)alkyl hexopyranosides, nonionic glycolipid mimetics, Carbohydr. Res., 2020, 488, 107905.

11-S. X. Song, M. L. Wu, X. P. He, Y. B. Zhou, L. Sheng, J. Li, G. R. Chen, The anomeric mixture of some $\mathrm{O}$-galactolipid derivatives is more toxic against cancer cells than either anomer alone, Bioorg. Med. Chem. Lett., 2012, 22, 2030-2032.

12-B. J. Boyd, C. J. Drummond, I. Krodkiewska, F. Grieser, How Chain Length, Headgroup Polymerization, and Anomeric Configuration
Govern the Thermotropic and Lyotropic Liquid Crystalline Phase Behavior and the Air-Water Interfacial Adsorption of Glucose-Based Surfactants, Langmuir, 2000, 16, 7359-7367.

13-A. Bilkova, E. Paulovicova, L. Paulovicova, M. Polakova, Antimicrobial activity of mannosederived glycosides, Monatsh. Chem., 2015, 146, 1707-1714.

14-C. Dias, A. Martins, A. Pelerito, M. C. Oliveira, M. Contino, N. A. Colabufo, A. P. Rauter, Assessing the Optimal Deoxygenation Pattern of Dodecyl Glycosides for Antimicrobial Activity Against Bacillus anthracis, Eur. J. Org. Chem., 2019, 2224-2233.

15-C. Dias, A. P. Rauter, 2-Deoxy glycosides as innovative antimicrobial and neuroprotective molecules, American Chemical Society, 2016, pp. ICS-158.

16-F. V. M. Silva, M. Goulart, J. Justino, A. Neves, F. Santos, J. Caio, S. Lucas, A. Newton, D. Sacoto, E. Barbosa, M. S. Santos, A. P. Rauter, Alkyl deoxy-arabino-hexopyranosides: Synthesis, surface properties, and biological activities, Bioorg. Med. Chem., 2008, 16, 4083-4092.

17- H. Luders, Synthesis of alkyl glucosides and alkyl polyglucosides, Surfactant Sci. Ser., 2000, 91, 19-75.

18-J. Nowicki, M. Moscipan, E. NowakowskaBogdan, J. Woch, Micellar Catalysis in Fischer Glycosidation: En Route to Diverse Functional Glycosides, ChemistrySelect, 2019, 4, 13841-13845.

19-W. Szeja, Glycosidation of sugars in the Fischer reaction, Wiad. Chem., 1979, 33, 7-23.

20-S. Traboni, E. Bedini, M. Giordano, A. Iadonisi, Three Solvent-Free Catalytic Approaches to the Acetal Functionalization of Carbohydrates and Their Applicability to One-Pot Generation of Orthogonally Protected Building Blocks, Adv. Synth. Catal., 2015, 357, 3562-3572.

21-S. N. Baytas, Q. Wang, N. A. Karst, J. S. Dordick, R. J. Linhardt, Solid-phase chemoenzymatic synthesis of C-sialosides, J. Org. Chem., 2004, 69, 6900-6903.

22-W. Huang, S. Groothuys, A. Heredia, B. H. M. Kuijpers, F. P. J. T. Rutjes, D. F. L. van, L. X. Wang, Enzymatic glycosylation of triazolelinked GlcNAc/Glc-peptides: synthesis, stability and anti-HIV activity of triazole-linked HIV-1 gp41 glycopeptide C34 analogues, Chembiochem, 2009, 10, 1234-1242.

23-S. Muthana, H. Cao, X. Chen, Recent progress in chemical and chemoenzymatic synthesis of carbohydrates, Curr. Opin. Chem. Biol., 2009, 13, 573-581.

24-E. L. Smith, J. P. Giddens, A. T. Iavarone, K. Godula, L. X. Wang, C. R. Bertozzi, Chemoenzymatic Fc Glycosylation via Engineered Aldehyde Tags, Bioconjugate Chem., 2014, 25, 788-795. 
25-H. Akita, K. Kurashima, T. Nakamura, K. Kato, Chemo-enzymatic syntheses of naturally occurring $\beta$-glucosides, Tetrahedron: Asymmetry, 1999, 10, 2429-2439.

26-P. Kovac, R. B. Taylor, C. P. J. Glaudemans, General synthesis of (1 fwdarw. 3)-.beta.-Dgalacto oligosaccharides and their methyl .beta.glycosides by a stepwise or a blockwise approach, J. Org. Chem., 1985, 50, 5323-5333.

27-W. Koenigs, E. Knorr, Über einige Derivate des Traubenzuckers und der Galactose, Ber. Dtsch. chem. Ges., 1901, 34, 957-981.

28-G. Capozzi, S. Menichetti, C. Nativi, Selective glycosidation reactions and their use in medicinal chemistry, Methods Princ. Med. Chem., 2000, 7 , 221-259.

29-P. J. Garegg, P. Konradsson, I. Kvarnstroem, T. Norberg, S. C. T. Svensson, B. Wigilius, Studies on Koenigs-Knorr glycosidations, Acta Chem. Scand., Ser. B, 1985, 39, 569-577.

30-K. Krohn, in Orgaic Synthesis Highlights (J. Mulzer, H.J. Altenbach, M. Braun, K. Krohn, H.U. Reissig, Eds), Synthesis of $O$-glycosides, VCH, Weinheim 1991, 277-285.

31-K. Nakayama, K. Higashi, T. Soga, K. Uoto, T. Kusama, Zinc triflate-promoted glycosidation: synthesis of lipid A disaccharide intermediates, Chem. Pharm. Bull., 1992, 40, 2909-2912.

32-Y. Singh, A. V. Demchenko, Defining the Scope of the Acid-Catalyzed Glycosidation of Glycosyl Bromides, Chem. Eur. J., 2019, 26, 1042-1051.

33-Z. Wimmer, L. Pechova, D. Saman, KoenigsKnorr synthesis of cycloalkyl glycosides, Molecules, 2004, 9, 902-912.

34-R. U. Lemieux, W. P. Shyluk, A new synthesis of $\beta$-glucopyranosides, Can. J. Chem., 1953, 31, 528-535.

35-H. Hönig, H. Weidmann, Simple synthesis of methyl- $\beta$-D-glucopyranoside, Synthesis, 1975, 804.

36-F. Dasgupta, P. P. Singh, H. C. Srivastava, Acetylation of carbohydrates using ferric chloride in acetic anhydride, Carbohydr. Res., 1980, 80, 346-349. https://doi.org/10.1016/S00086215(00)84876-4.

37-C. H. Hamann, H. Polligkeit, P. Wolf, Z. Smiatacz, An electrochemical synthesis of methyl $\alpha$-isomaltoside, Carbohydr. Res., 1994, $265,1-7$.

38-M. L. Wolfrom, A. B. Diwadkar, J. Gelas, D. Horton, New method of acetonation. Synthesis of 4,6- $O$-isopropylidene-Dglucopyranose, Carbohyd. Res., 1974, 35, 87-96.

39-G. Hodosi, P. Kovac, Manipulation of free carbohydrates via stannylene acetals. Preparation of $\beta$-per- $O$-acyl derivatives of D-mannose, L-rhamnose, 6-O-trityl-D-talose, and D-lyxose, Carbohydr. Res., 1997, 303, 239-243.

40-Z. Zhang, G. Magnusson, Conversion of p-methoxyphenyl glycosides into the corresponding glycosyl chlorides and bromides, and into thiophenyl glycosides, Carbohydr. Res., 1996, 295, 41-55.

41-J. N. Schumacher, Isolation of 6- $O$-acetyl-2,3,4tri- $O$-[(+)-3-methylvaleryl]- $\beta$-D-glucopyranose from tobacco, Carbohyd. Res., 1970, 13, 1-8.

42-A. V. Wartburg, E. Angliker, J. Renz, Lignanglucoside aus Podophyllum peltatum L. 7. Mitteilung über mitosehemmende Naturstoffe, Helv. Chim. Acta, 1957, 40, 1331-1357.

43-P. M. L. Goncalves, S. M. Roberts, P. W. H. Wan, Regioselective acylation of carbohydrate derivatives using lipases leading to a facile twostep procedure for the separation of some $\alpha$ - and $\beta$-glucopyranosides and galactopyranosides, Tetrahedron, 2004, 60, 927-932.

44-S. Veibel, F. Eriksen, Synthesis and properties of several $\beta$-alkyl glucosides, Bull. Soc. Chim. Fr., Mem., 1936, 3, 277-283.

45-H. L. Yu, J. H. Xu, W. Y. Lu, G. Q. Lin, Identification, purification and characterization of $\beta$-glucosidase from apple seed as a novel catalyst for synthesis of $O$-glucosides, Enzyme Microb. Technol., 2007, 40, 354-361.

46-W. Pigman, R. O. Laffre, The preparation of butyl $\alpha$-D-glucoside, J. Am. Chem. Soc., 1951, 73, 4994-4995.

47-M. Kishida, M. Nishiuchi, K. Kato, H. Akita, Chemoenzymatic synthesis of n-hexyl and $O-\beta$ D-xylopyranosyl-( $1 \rightarrow 6)-\beta$-D-glucopyranosides, Chem. Pharm. Bull., 2004, 52, 1105-1108.

48-V. Y. Joshi, M. R. Sawant, A convenient stereoselective synthesis of $\beta$-Dglucopyranosides, Indian J. Chem., Sect. B, 2006, 45, 461-465.

49-H. Akita, E. Kawahara, K. Kato, Chemoenzymatic synthesis of rhodiooctanoside isolated from Chinese medicines, Rhodiola radix, Tetrahedron: Asymmetry, 2004, 15, 1623-1629.

50-C. R. Noller, W. C. Rockwell, Preparation of some higher alkyl glucosides, J. Am. Chem. Soc., 1938, 60, 2076-2077.

51-X. Li, J. Turanek, P. Knoetigova, H. Kudlackova, J. Masek, D.B. Pennington, S.E. Rankin, B.L. Knutson, H.-J. Lehmler, Synthesis and biocompatibility evaluation of fluorinated, single-tailed glucopyranoside surfactants, New J. Chem., 2008, 32, 2169-2179.

52-R. Hori, Y. Ikegami, Studies on carbohydrate derivatives. V. Syntheses of alkyl galactosides and alkyl glucosides, Yakugaku Zasshi, 1959, 79, 80-83. https://doi.org/10.1248/yakushi1947.79.1_80.

53-M. Prostenik, N. Krvavica, The sphingolipide series. VIII. Synthesis of $N$-benzoylsphingine glucosides, compounds related to cerebrosides, Croat. Chem. Acta, 1957, 29, 101-105.

54-W. Korytnyk, J. A. Mills, 128. Preparation and properties of some poly- $O$-acetylglycosyl chlorides and the "unstable" series, J. Chem. Soc., 1959, 636-649. 
55-C. S. Hudson, J. K. Dale, The isomeric pentaacetates of mannose, J. Am. Chem. Soc., 1915, 37, 1280-1282.

56-C. Mayato, R. Dorta, J. Vazquez, Experimental evidence on the hydroxymethyl group conformation in alkyl $\beta$-mannopyranosides, Tetrahedron: Asymmetry, 2004, 15, 2385-2397.

57-G. Zemplen, A. Gerecs, T. Valatin, Levomannosan, Ber. Dtsch. Chem. Ges. B, 1940, 73B, 575-580.

58-V. Deulofeu, J. O. Deferrari, The reaction of ammonia with some acetylated and benzoylated monosaccharides. III. Derivatives of D-galactose, J. Org. Chem., 1952, 17, 1097-1101.

59-R. K. Ness, H. G. Fletcher, J. C. S. Hudson, 1,4-Anhydro-D-galactitol, J. Am. Chem. Soc., 1951, 73, 3742-3744.

60-Z. Liu, H. S. Byun, R. Bittman, Synthesis of Immunostimulatory $\alpha$ - $C$-Galactosylceramide Glycolipids via Sonogashira Coupling,

Asymmetric Epoxidation, and Trichloroacetimidate-Mediated Epoxide Opening, Org. Lett., 2010, 12, 2974-2977.

61-X. Sun, L. Ji, S. Ren, S. Wan, T. Jiang, Chemical Synthesis and In Vitro Antitumor Activity of Quinizarin Glycoside Analogs, Synth. Commun., 2008, 38, 4116-4124.

62-G. J. L. Bernardes, E. J. Grayson, S. Thompson, J. M. Chalker, J. C. Errey, F. ElOualid, T. D. W. Claridge, B. G. Davis, From disulfide- to thioether-linked glycoproteins, Angew. Chem., Int. Ed., 2008, 47, 2244-2247.

63-A. G. Goncalves, M. D. Noseda, M. E. R. Duarte, T. B. Grindley, Regioselective synthesis of longchain ethers and their sulfates derived from methyl $\beta$-D-galactopyranoside and derivatives via dibutylstannylene acetal intermediates, Carbohydr. Res., 2005, 340, 2245-2250.

64-L. J. J. Hronowski, W. A. Szarek, G. W. Hay, A. Krebs, W. T. Depew, Synthesis and characterization of $1-O$ - $\beta$-lactosyl- $(R, S)$-glycerols and 1,3-di- $O$ - $\beta$-lactosylglycerol, Carbohydr. Res., 1989, 190, 203-218.

65-R. Sardzik, G. T. Noble, M. J. Weissenborn, A. Martin, S. J. Webb, S. L. Flitsch, Preparation of aminoethyl glycosides for glycoconjugation, Beilstein J. Org. Chem., 2010, 6 699-703.

66-H. Brunne, C. Keck, Enantioselective catalysis. 157. Carbohdrate-based, water-soluble ligands for the stereoselective hydrogenation of folic acid, Z. Anorg. Allg. Chem., 2005, 631, 2555-2562.

67-F. Smith, . W. Van Cleve, A reinvestigation of the preparatin of $\beta$-methyl lactoside, J. Am. Chem. Soc., 952, 74, 1912-1913.

68-M. A. Ali,L. Hough, A. C. Richardson, Thio and epidithio deriatives of methyl $\beta$-lactoside, Carbohydr. Rs., 1991, 216, 271-287. 